\title{
Silencing of CKIP-1 promotes tumor proliferation and cell adhesion-mediated drug resistance via regulating AKT activity in non-Hodgkin's lymphoma
}

\author{
XINHUA ZHU ${ }^{1 *}$, YU OUYANG ${ }^{3 *}$, FEI ZHONG ${ }^{3}$, QIRU WANG $^{3}$, LINLIN DING $^{3}$, \\ PEIPEI ZHANG ${ }^{3}$, LINGLING CHEN ${ }^{2}, \mathrm{HONG} \mathrm{LIU}^{2}$ and SONG HE ${ }^{1}$ \\ ${ }^{1}$ Department of Pathology, Affiliated Cancer Hospital of Nantong University, Nantong, Jiangsu 226361; \\ ${ }^{2}$ Department of Hematology, Affiliated Hospital of Nantong University, Nantong University, Nantong, Jiangsu 22600; \\ ${ }^{3}$ Jiangsu Province Key Laboratory for Inflammation and Molecular Drug Target, \\ Nantong University, Nantong, Jiangsu 226001, P.R. China
}

Received May 23, 2016; Accepted October 27, 2016

DOI: $10.3892 /$ or.2016.5233

\begin{abstract}
Casein kinase 2 interacting protein-1 (CKIP-1; also known as PLEKHO1) is involved in regulating many processes such as cell proliferation, differentiation and apoptosis. CKIP-1 also plays an important role in many types of cancer, such as colon, breast cancer and human osteosarcoma. In the present study, we found that CKIP-1 was reversely associated with the proliferation of non-Hodgkin's lymphoma (NHL) and cell adhesion mediated drug resistance (CAM-DR). We demonstrated that knockdown of CKIP-1 promoted the proliferation of NHL cells through interacting with Akt and suppressing Akt phosphorylation. In addition, adhesion of lymphoma cells to fibronectin or stroma cells (HS-5 cells) decreased CKIP-1 expression, which led to the upregulation of Akt phosphorylation. Importantly, we showed that the phosphorylation of Akt was correlated with CAM-DR phenotype in NHL cells. Taken together, the present study shed new light on the molecular mechanism of CAM-DR in NHL and targeting CKIP-1 may be a novel therapeutic target for NHL.
\end{abstract}

Correspondence to: Dr Hong Liu, Department of Hematology, Affiliated Hospital of Nantong University, Nantong University, Nantong, Jiangsu 22600, P.R. China

E-mail: hongliu63@163.com

Dr Song He, Department of Pathology, Affiliated Cancer Hospital of Nantong University, 30 North Tongyang Road, Pingchao, Nantong, Jiangsu 226361, P.R. China

E-mail: hesongnt@126.com

*Contributed equally

Abbreviations: NHL, non-Hodgkin's lymphoma; FBS, fetal bovine serum; CAM-DR, cell adhesion mediated drug resistance

Key words: non-Hodgkin's lymphoma, CKIP-1, proliferation, cell adhesion resistance, Akt

\section{Introduction}

Non-Hodgkin's lymphomas (NHLs), derived from B- or T-cell, constitute a heterogeneous group of malignant lymphoproliferative disorders $(1,2)$, which accounts for approximately $90 \%$ of all lymphomas (3). The incidence of NHLs has increased in recent years and is still associated with significant mortality. Despite intensive efforts in developing new therapies, the emergence of clinical drug resistance remains a barrier to successful treatment of lymphoma $(4,5)$. Recently, evidence has accumulated showing that lymphoma tumor microenvironment provides sanctuary for lymphoma cells through interaction which occurs between the lymphoma cell and its microenvironment (lymph node stroma) (6). Adhesion of lymphoma cells to microenvironment components is shown to be a critical determinant for mediating tumor resistance to cytotoxic therapy (cell adhesion mediated drug resistance; CAM-DR) (7). CAM-DR is one of the most important mechanisms in tumor cell microenvironment protection. However, it remains unclear how the lymphoma microenvironment influences lymphoma cell survival and responses to therapy, as well as the molecular mechanisms involved.

The PH domain-containing casein kinase 2 interacting protein-1 (CKIP-1; also known as PLEKHO1) is involved in regulating many processes such as cell proliferation, differentiation and apoptosis (8). CKIP-1 also plays an important role in many cancers, such as colon, breast cancer and human osteosarcoma (9). CKIP-1 has been reported to regulate numerous important proteins controlling cell survival, such as CK2, Akt and Smurf1. It has been generally demonstrated that the activities of CK2, Akt and Smurf1 are elevated in many human cancers (10-12). In addition, CKIP-1 negatively regulates several important pathways, such as TGF- $\beta /$ BMP signaling, and PI3K/Akt signaling, indicating that CKIP-1 acts as an crucial master in tumorigenicity control (11). A previous study reported that CKIP-1 enhances sensitivity to chemotherapy drugs by targeting the Akt $\mathrm{PH}$ domain and suppressing Akt kinase activity in human cancers (10). It has been reported that knockdown of CKIP-1 in colon cancer 
cells shortened the G1/S phase and reduced cell proliferation due to decreased Akt kinase activity (13). These observations together revealed that CKIP-1 might function as a potential tumor suppressor. However, the functional role of CKIP-1 in non-Hodgkin's lymphoma (NHL) has never been elucidated. Considering the pivotal role CKIP-1 played in tumorigenicity control and the fact that CKIP-1 can regulate Akt activity, it would be intriguing to verify whether CKIP-1 plays a key role in CAM-DR in NHL.

In the present study, we aimed to investigate the expression and function of CKIP-1 in NHL and CAM-DR phenotype. This study demonstrated for the first time that CKIP-1 is a novel prognostic indicator in NHL. Furthermore, we also demonstrated that CKIP-1 plays a critical role in CAM-DR via interacting with Akt and regulating Akt kinase activity in NHL. Taken together, this study may provide a novel perspective for a better understanding of the role for CKIP-1in NHL and CAM-DR.

\section{Materials and methods}

Cell culture. Burkitt lymphoma cell-line Raji and diffuse large B-cell lymphoma (DLBCL) cell lines OCI-Ly8 were obtained from Fudan University (Shanghai, China). Bone marrow stromal cell line HS-5 was obtained from Cell Library, China Academy of Science. The human lymphoma cell lines were grown in suspension in RPMI-1640 (Sigma-Aldrich, Rehovot, Israel) and the HS-5 was grown in F12 (Sigma-Aldrich), supplemented with $10 \%$ fetal bovine serum (FBS; Gibco-BRL, Grand Island, $\mathrm{NY}, \mathrm{USA}$ ) at $37^{\circ} \mathrm{C}$ and $5 \% \mathrm{CO}_{2}$.

Cell co-culture. Firstly, dishes were coated with $40 \mathrm{lg} / \mathrm{ml}$ human FN (Sigma-Aldrich) or HS-5 cells at $37^{\circ} \mathrm{C}$. Secondly, lymphoma cells $\left(10^{5} \mathrm{cells} / \mathrm{ml}\right)$ were allowed to adhere to preincubated FN or HS-5 for $24 \mathrm{~h}$. Finally, adherent cells were removed for subsequent experiments.

Western blot analysis and antibodies. The following monoclonal antibodies were purchased: CKIP-1 (Cell Signaling Technology, Beverly, MA, USA); AKT (Cell Signaling Technology), Gsk-3 $\beta$ (Cell Signaling Technology), anti-p-Akt (Ser473) and anti-pAkt (Thr308) (Cell Signaling Technology) and pGsk-3 $\beta$ (Cell Signaling Technology); anti- $\beta$-actin (Sigma-Aldrich). Protein was run on a $10 \%$ PAGE and transferred to polyvinylidine difluoride filter (PVDF) membranes. The membranes were blocked with phosphate-buffered saline (PBS) containing 5\% skim milk and $0.1 \%$ Tween-20 and then incubated with primary antibody overnight at $4^{\circ} \mathrm{C}$. After washing with PBS containing $0.1 \%$ Tween-20 three times, each for $5 \mathrm{~min}$, filters were incubated with horseradish peroxidaseconjugated secondary antibody for $2 \mathrm{~h}$ at room temperature. Values were responsible for at least three independent experiments.

Transient transfection. Full-length CKIP-1 cDNA were amplified using PCR and subcloned into pCMV-HA and pcDNA3.1 constructs. Control-siRNA and CKIP-1-siRNA were purchased from Shanghai GenePharma, Co., Ltd. (Shanghai, China). Transfection was performed using Lipofectamine 2000 transfection reagent (Invitrogen) according to the manufacturer's instructions. Then, siRNA-resistant CKIP-1 construct (designated myc-rCKIP-1) was generated by introducing silent mutations in the targeting regions for CKIP-1-siRNA. NHL cells were plated in FBS free RPMI-1640 medium without antibiotics and cells were transfected at $70-90 \%$ confluence. Transfection was carried out according to the manufacturer's instructions. Transfected cells were used for subsequent experiments $48 \mathrm{~h}$ after transfection.

RNA isolation and reverse transcription-polymerase chain reaction (RT-PCR). Total RNA of Raji cells and OCI-Ly8 cells were extracted by a TRIzol extraction kit according to the manufacturer's protocol. The total RNA was reverse-transcribed by the ThermoScript RT-PCR system (Invitrogen, Carlsbad, CA, USA). cDNA was amplified using the following primers: CKIP-1 F, 5'-AATTCTGCGGGAAAGGGATTT-3' and R, 5'-AACACCTCCTGACTGTTTTCTC-3'; c-Myc F, 5'-AATCCTGTACCTCGTCCGAT-3' and R, 5'-TCTTCTC CACAGACACCACA-3'; cyclin D1 F, 5'-TGCTACCGCACA ACGCA-3' and R, 5'-TCAATCTGTTCCTGGCAGGC-3'; cyclin E F, 5'-CTCCAGGAAGAGGAAGGCAA-3' and R, 5'-TCGATTTTGGCCATTTCTTCA-3'; GAPDH F, 5'-AGG TCGGTGTGAACGGATTTG-3' and R, 5'-TGTAGACCATG TAGTTGAGGTCA-3'. After amplification, the products were separated on an agarose (1.5\%) gel and visualized under UV light.

Cell viability assay. The cells were seeded on a 96-well plate (Corning Incorporated, Corning, NY, USA) with a density of $1 \times 10^{6} /$ well in volumes of $100 \mu \mathrm{l}$ and grew $12 \mathrm{~h}$ with or without the addition of doxorubicin (Sigma-Aldrich). Then, cell proliferation was measured using Cell Counting kit-8 (CCK-8; Dojindo Laboratories, Kumamoto, Japan). The absorbance was read by a fluorometer (CytoFluor; Applied Biosystems, Foster City, CA, USA) at $450 \mathrm{~nm}$ with a reference wavelength of $630 \mathrm{~nm}$.

Apoptosis measurements. Drug-induced apoptosis following exposure to doxorubicin (Sigma-Aldrich) was detected in NHL cells. In short, following $48 \mathrm{~h}$ of chemotherapy agent exposure, apoptotic cells were detected using Annexin V-FLUOS Staining kit (Roche Diagnostics, Indianapolis, IN, USA) according to the manufacturer's protocol. Cells were washed three times and were resuspended in 1001 of Annexin V-FLUOS labeling solution at a concentration of $1 \times 10^{6}$ cells $/ \mathrm{ml}$ incubate in the dark for $15 \mathrm{~min}$. To analyse apoptosis, flow cytometry (BD FACSAriaII) was performed to analyze the labeled cells.

Immunoprecipitation. Immunoprecipitation was performed as previously described (14). In short, cell lysates was incubated with anti-CKIP-1 antibody or control IgG (Santa Cruz Biotechnology, Santa Cruz, CA, USA) at $4^{\circ} \mathrm{C}$ overnight. Protein A/G (Sigma-Aldrich) was then added for $2 \mathrm{~h}$ at $4^{\circ} \mathrm{C}$ with rocking. The precipitates were washed three times with homogenization buffer and boiled for $10 \mathrm{~min}$ with SDS sample buffer followed by western blot analysis.

Soft agar colony assays. In short, cells were resuspended at $1 \times 10^{3}$ cells in $0.5 \mathrm{ml}$ of $0.35 \%$ agar solution (Sigma-Aldrich, St. Louis, MO, USA) containing RPMI-1640 cell culture 



C



Raji
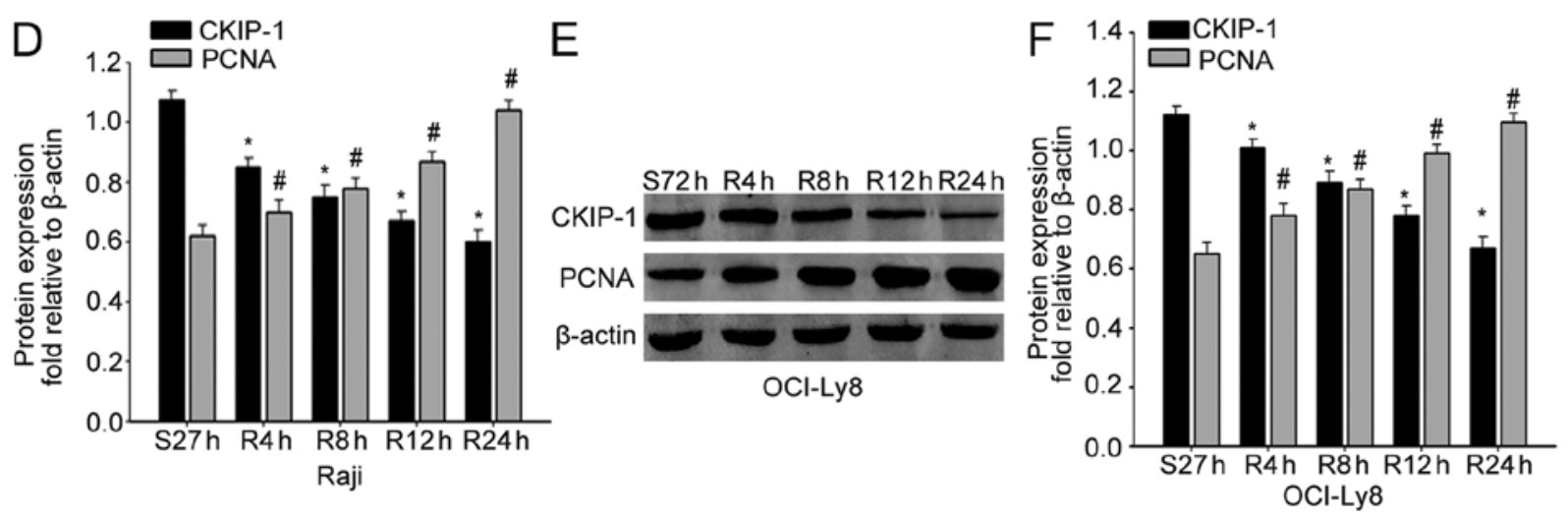

Figure 1. CKIP-1 expression was downregulated in proliferating NHL cells. (A and B) Flow cytometry was used to analyze the cell cycle progression of Raji cells or OCI-Ly8 cells, which were subjected to serum starvation (S) for $72 \mathrm{~h}$ and re-feeding (R) for 0, 4, 8, 12 and $24 \mathrm{~h}$. (C and E) Western blot analysis showed that the expression of CKIP- 1 and PCNA that were subjected to serum starvation for $72 \mathrm{~h}$ and re-feeding for $0,4,8,12$ and $24 \mathrm{~h}$. $\beta$-actin was used as a control for protein load and integrity. (D and F) A bar chart demonstrated the ratio of CKIP-1 and PCNA expression to $\beta$-actin at different times. Data are presented as mean \pm SEM of three independent measurements $\left({ }^{*}, \mathrm{P}<0.05\right.$ compared with $\left.\mathrm{S} 72 \mathrm{~h}\right)$.

medium and layered on top of a $0.8 \%$ agar layer in 24 -well plates. Plates were then maintained for 14 days at $37^{\circ} \mathrm{C}$ with $5 \% \mathrm{CO}_{2}$. Cell colonies were stained with $0.5 \%$ crystal violet and visualized by microscopy.

Statistical analysis. All experiments were repeated at least three times. All data are reported as mean \pm SEM. The calculations were analyzed using the Statistical Package for Social Science SPSS 19.0 software (SPSS, Inc., Chicago, IL, USA). Statistical significance was set at $\mathrm{P}<0.05$.

\section{Results}

CKIP-1 expression levels were deregulated in proliferating $N H L$. It has been reported that CKIP-1 is deregulated in multiple malignant tumor cell lines and tissues, such as breast (15), colon cancer (11) and human osteosarcoma (16). However, the expression of CKIP-1 in NHL is still unknown. In the present study, we first investigated the alterations of CKIP-1 during NHL progression. Raji and OCI-Ly8 cells were cultured in serum-free medium for $72 \mathrm{~h}$, and released with RPMI-1640 medium containing 10\% FBS for the indicated period of time. Flow cytometric analysis demonstrated that cells were arrested in the G1 phase due to serum deprivation, and the cells were released from the G1 phase and entered the $\mathrm{S}$ phase following serum re-feeding (Fig. 1A and B). Next, we analyzed the expression of CKIP-1 in proliferating NHL cells.
As expected, CKIP-1 levels were decreased gradually after serum stimulation, which was in accordance with upregulation of PCNA in both Raji and OCI-Ly8 cells (Fig. 1C-F). These data indicated that CKIP-1 might promote proliferation of NHL cells.

Knockdown of CKIP-1 promotes cell proliferation. The repressed CKIP-1 expression in the progression of NHL inspired us to investigate whether CKIP-1 functions as a candidate tumor suppressor. Thus, we asked whether CKIP-1 could also have a role in regulation of NHL cell proliferation. To confirm our assumption, OCI-Ly8 and Raji cells were transfected with CKIP-1-siRNA or myc-tagged CKIP-1 (myc-CKIP-1) or their respective control. The interference efficiency was confirmed by western blot analyses (Fig. 2A and B). CCK-8 assays were used to investigate the effect of CKIP-1 on OCI-ly8 and Raji cell growth. We observed that cells were transfected with myc-CKIP-1 showed reduced cell growth. On the contrary, cells were transfected with CKIP1-siRNA exhibited increased cell growth (Fig. 2C and D). In addition, colony formation assay showed that knockdown of CKIP-1 resulted in a significant promotion of cell growth (Fig. 2E and F). All the data suggested that CKIP-1 might play a role in regulating cell proliferation.

Knockdown of CKIP-1 promotes cell cycle progression. To further confirm the effect of CKIP-1 on cell cycle regulation, 
A


E



B
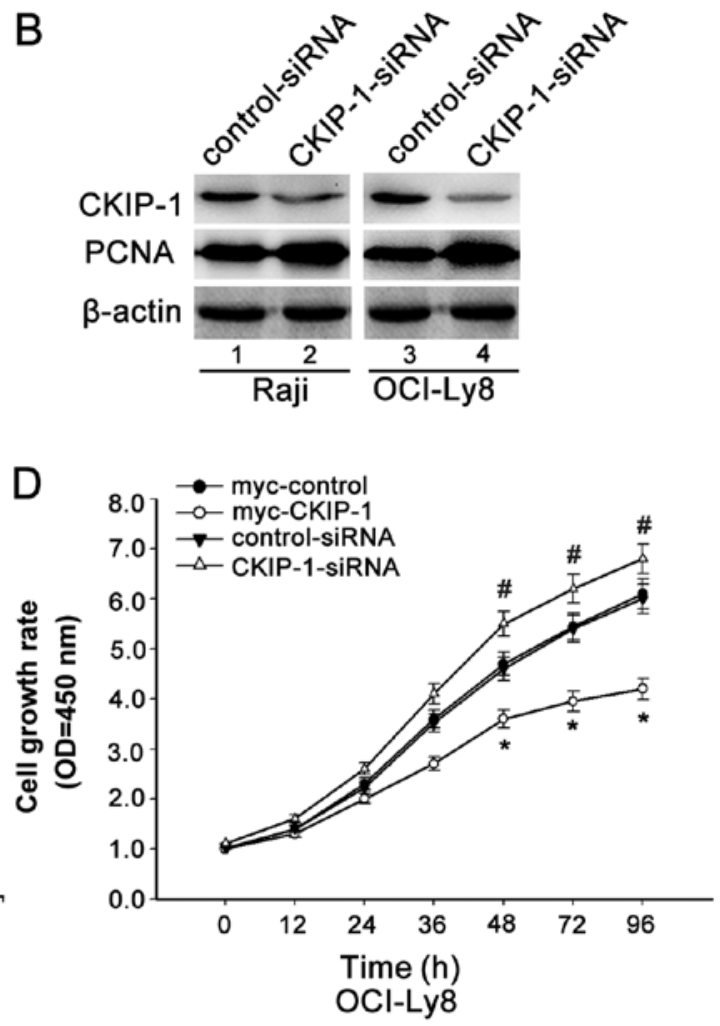

F

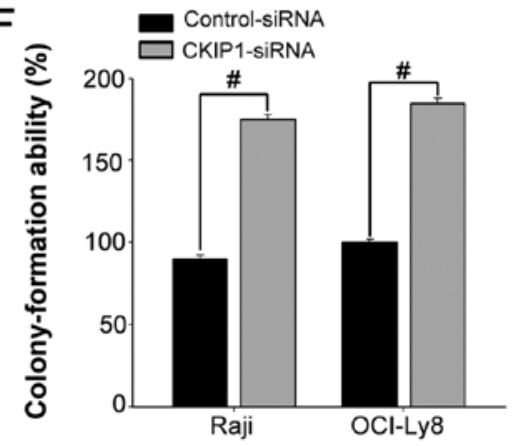

Figure 2. Knockdown of CKIP-1 promotes cell proliferation via modulating cell cycle progression. (A and B) OCI-Ly8 and Raji cells were transfected with myc-CKIP-1, CKIP-1-siRNA or their respective control. The interference efficiencies were confirmed by western blot analysis. (C and D) OCI-Ly8 and Raji cells were transfected with myc-CKIP-1, CKIP-1-siRNA or their respective control. CCK-8 assay was then performed to evaluate the cell growth. Data are presented as mean \pm SEM of three independent measurements $\left({ }^{*}, \mathrm{P}<0.05\right)$. (E and F) OCI-Ly8 and Raji cells were transfected with either CKIP-1-siRNA or control-siRNA. Soft agar colony formation analysis was performed to evaluate the effect of CKIP-1 on NHL cells proliferation. Data are presented as mean \pm SEM of three independent measurements $(*, \# \mathrm{P}<0.05)$.

flow cytometric analysis was conducted and showed that CKIP-1 generate a decrease in the percentage of cells in the G1 phase when OCI-Ly8 and Raji cells were transfected with CKIP-1-siRNA, but more cells were found in the G1 phase in the presence of myc-CKIP-1 (Fig. 3A and B). To further understand the effect of CKIP-1 on cell cycle progression, we measured the cell cycle-regulatory protein expression levels in NHL. RT-PCR revealed that cells were transfected with CKIP-1-siRNA expressed more c-Myc, cyclin D1 and cyclin E compared with control-siRNA transfected ones (Fig. 3C). Moreover, western blot analysis showed that knockdown of CKIP-1 resulted in a significant increase of c-Myc, cyclin D1, cyclin E and PCNA expression, whereas overexpression of CKIP-1 resulted in a significant decrease of their expression (Fig. 3D). All the data indicate that knockdown of CKIP-1 in NHL cells promotes cell cycle progression.
CKIP-1 reverses CAM-DR in NHL. A previous study reported that CKIP-1 is correlated to chemotherapy in human cancer cells $(8,10,11)$. To determine whether CKIP-1 was associated with CAM-DR in NHL, cell adhesion assays were first performed to assess the expression of CKIP-1. Western blot analysis and RT-PCR analysis showed that CKIP-1 expression was less obvious when cells adhered to FN or HS-5 cells compared with those cultured in suspension (Fig. 4A and B). To confirm whether CKIP-1 had an effect on CAM-DR in NHL, we prepared myc-tagged plasmid expressing CKIP-1, which is resistant to silencing by CKIP-1-siRNA (designated myc-rCKIP-1). The transfection efficiencies were confirmed by western blot analysis (Fig. 4C). Then, Raji and OCI-Ly8 cells were treated with $1 \mu \mathrm{M}$ doxorubicin (Doxo). CCK-8 assays showed that adhesion to FN or HS-5 cells significantly protected OCI-Ly8 and Raji cells from chemotherapeutics 

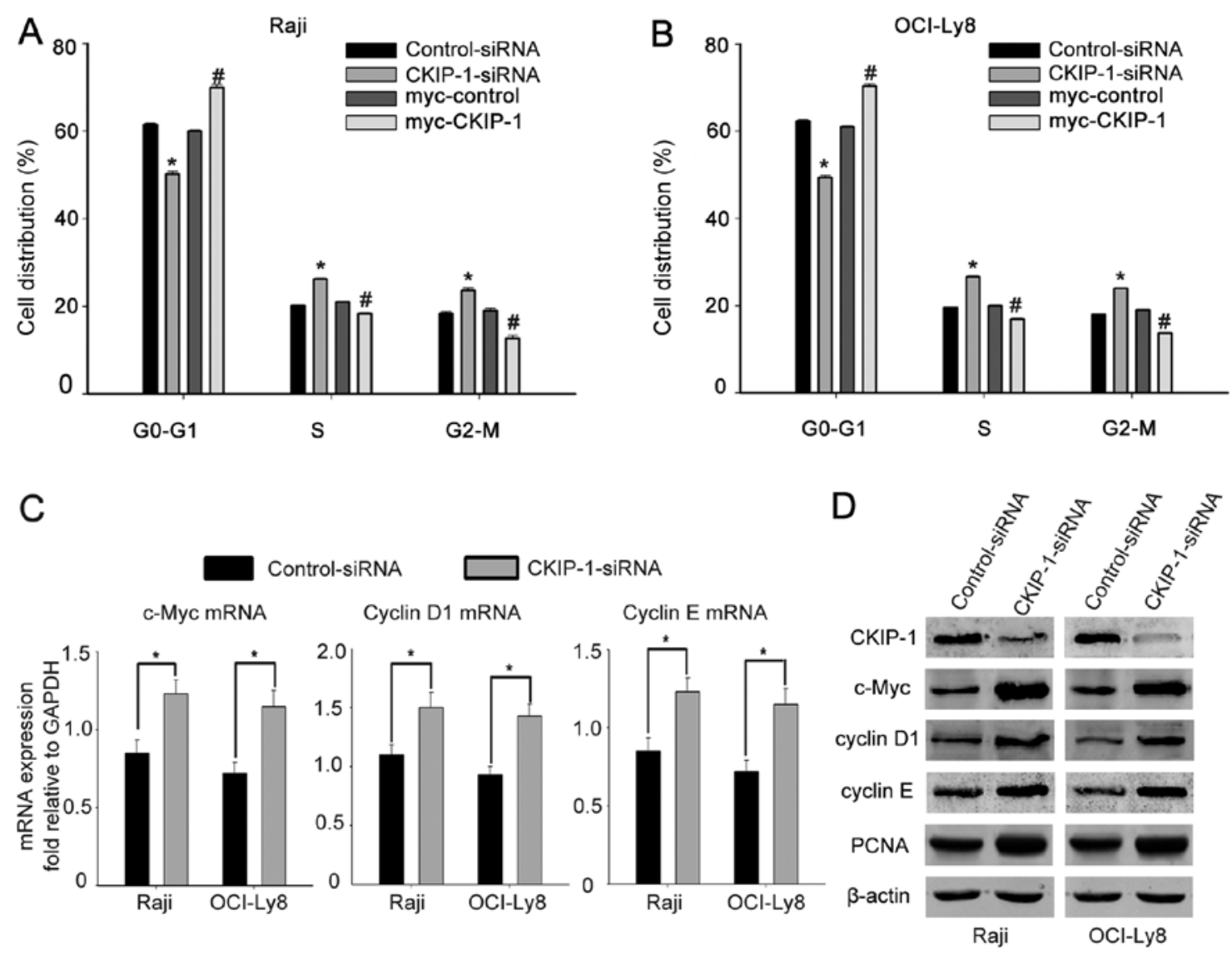

Figure 3. Knockdown of CKIP-1 inhibits cell cycle progression. (A and B) OCI-Ly8 and Raji cells were transfected with either CKIP-1-siRNA or myc-CKIP-1 or their respective control. Flow cytometric analysis was performed to analyze the cell cycle distribution. Data are presented as mean \pm SEM of three independent measurements $\left({ }^{* \#} \mathrm{P}<0.05\right)$. (C) OCI-Ly8 and Raji cells were transfected with CKIP-1-siRNA or control-siRNA. Quantitative PCR analysis of the expression levels of cell cycle-related genes c-Myc, cyclin D1 and cyclin E. Data are presented relative to the expression of GAPDH (mean \pm SEM). ${ }^{*}<<0.05$. (D) OCI-Ly8 and Raji cells were transfected with either CKIP-1-siRNA or control-siRNA. Western blot assays were performed to analyze the expression of CKIP-1, c-Myc, cyclin D1, cyclin E and PCNA.

compared to those cultured in suspension. In addition, we found that this protective effect was increased in cells transfected with CKIP-1-siRNA. Notably, presentation of the myc-rCKIP-1 in CKIP-1-depleted cells abrogated the protective effect (Fig. 4D). Subsequently, flow cytometric analysis also showed that knockdown of CKIP-1 in Raji and OCI-Ly8 cells adhered to FN or HS-5 cells significantly decreased cell apoptosis. On the contrary, the presentation of the mycrCKIP-1 in CKIP-1-depleted cells increased cell apoptosis (Fig. 4E). These data suggested that overexpression of CKIP-1 could reverse CAM-DR in NHL.

CKIP-1 negatively regulates the PI3K/Akt pathway by interacting with Akt. A previous study demonstrated that CKIP-1 interacts with Akt, inhibits Akt phosphorylation and negatively regulates the PI3K/Akt pathway in breast cancer cells (15). Thus, we wondered whether CKIP-1 overcame CAM-DR phenotype by regulating PI3K/Akt pathway. Firstly, we sought to determine whether CKIP-1 could interact with Akt in NHL. The immunoprecipitation experiment showed that Akt could interact with CKIP-1 in OCI-Ly8 (Fig. 5A). Subsequently, Raji and OCI-Ly8 cells were transfected myc-CKIP-1 in different doses. Western blot analysis revealed that cells transfected with myc-CKIP-1 markedly reduced the phosphorylated Akt levels at Thr308 and Ser473 in a dose-dependent fashion.
Consistent with the decrease in phosphorylated Akt levels, cells expressing myc-CKIP-1 decreased the phosphorylation of GSK-3 $\beta$ in a dose-dependent fashion (Fig. 5B). Then, we used the Akt inhibitor (MK2206) to check the role of Akt in the proliferative effect of CKIP-1. We found that MK2206 significantly reduced p-Akt and p-GSK-3 $\beta$ expression in Raji and OCI-Ly8 cells (Fig. 5C). Notably, promotion of cell proliferation mediated by CKIP-1 was reversed by MK2206 in both Raji and OCI-Ly8 cells (Fig. 5D). Flow cytometric assays were used to investigate the effect of CKIP-1 on apoptosis. This showed that there were higher proportions of apoptotic cells in CKIP-1-siRNA-transfected cells compared with controlsiRNA transfected ones. Moreover, CKIP-1-siRNA-mediated apoptosis was increased in cells treated with MK2206. As expected, overexpression of CKIP-1 reduced apoptosis; however, anti-apoptotic effects of CKIP-1 were abrogated in MK2206-treated cells (Fig. 5E). All these data suggested that knockdown of CKIP-1 promoted cell proliferation and inhibited apoptosis by enhancing phospho-Akt expression.

CKIP-1 reverses CAM-DR via the Akt pathway. Previous data suggested that CKIP-1 played an important role in the CAM-DR phenotype. It has been reported that the Akt signaling is the major pathway controlling cell growth and survival, and elevated phosphorylated Akt levels could potentially be causative for 
A
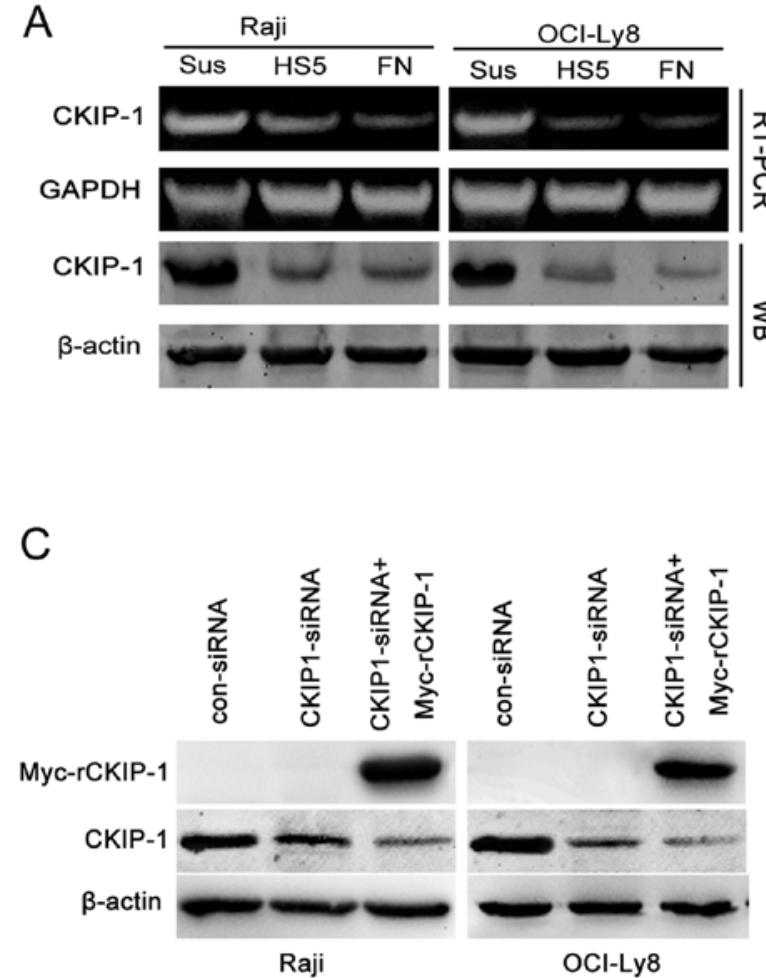
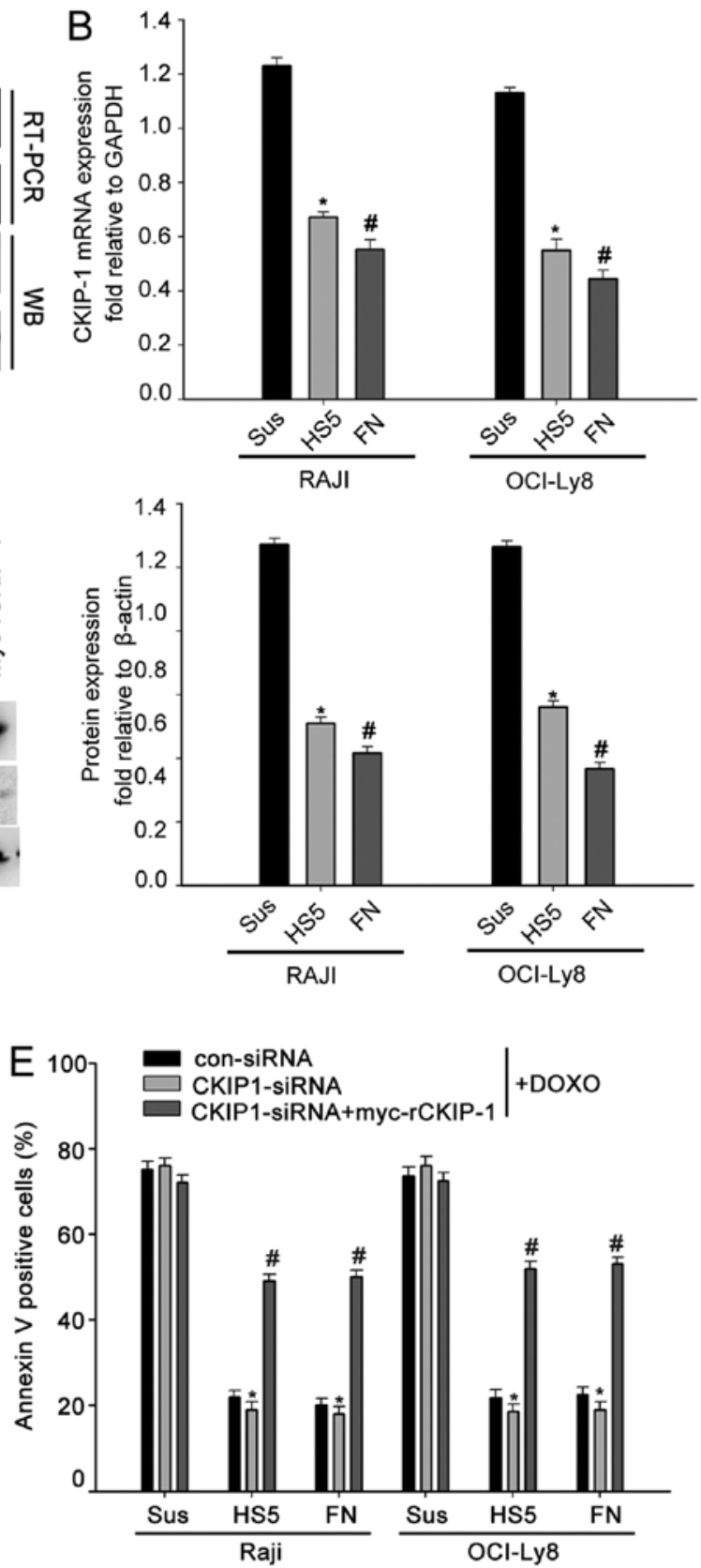

Figure 4. CKIP-1 reverses CAM-DR in NHL. (A and B) Raji and OCI-Ly8 cells were cultured in suspension or adhered to FN or HS-5 cells. Cells were analyzed for CKIP-1 and $\beta$-actin expression by western blot analysis. Total RNAs were analyzed by RT-PCR using CKIP-1-specific or GAPDH-specific primers. (C) Raji and OCI-Ly8 cells were transfected with control-siRNA, CKIP-1-siRNA or co-transfected with CKIP-1-siRNA and myc-tagged siRNA-resistant CKIP-1 (designated myc-rCKIP-1-1). Raji and OCI-Ly8 cells were analyzed for CKIP-1 and $\beta$-actin expression by western blot analysis. (D) Raji and OCI-Ly8 cells were transfected with control-siRNA, CKIP-1-siRNA or co-transfected with CKIP-1-siRNA and GFP-rCKIP-1, then cells were adhered to FN, HS-5 cells or cultured in suspension along with addition of $1 \mu \mathrm{M}$ doxorubicin (Doxo). Then, CCK-8 assays were performed to determine the cell viability. Data are presented as mean \pm SEM of three independent measurements $\left({ }^{*}, \#<0.05\right)$. (E) Raji and OCI-Ly8 cells were transfected with control-siRNA, CKIP-1-siRNA or co-transfected with CKIP-1-siRNA and GFP-rCKIP-1, Raji and OCI-Ly8 cells were then adhered to FN, HS-5 cells or cultured in suspension together with addition of $1 \mu \mathrm{M}$ Doxo. Flow cytometric analysis was used to evaluate Doxo-induced cell apoptosis. Data are presented as mean \pm SEM of three independent measurements $\left({ }^{*}, \#<0.05\right)$.

resistance to chemotherapy (17-19). Thus, we verified the role of Akt in cell adhesion model. Western blot analysis showed that the phosphorylation levels of Akt (Ser473 and Thr308) was more obvious in cells adhered to FN or HS-5 cells than cells cultured in suspension, suggesting that Akt signaling pathways promoted drug resistance (Fig. 6A). Thus, we suspected that CKIP-1 affected the CAM-DR by regulating the activity of Akt signaling pathways. Then, to determine whether CKIP-1 regulated CAM-DR phenotype via the Akt pathway, Raji and OCI-Ly8 cells were treated with MK2206 or DMSO (control). CCK-8 assays showed that knockdown of CKIP-1 in OCI-Ly8 and Daudi cell adhesion to FN or HS-5 cells significantly increased cell viability, but the protective effect mediated by knockdown of CKIP-1 was clearly reversed in MK2206-treated cells (Fig. 6B and C). All these data indicated that CKIP reversed CAM-DR via Akt pathway. 
A

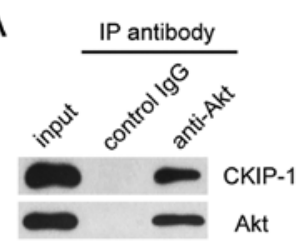

Akt

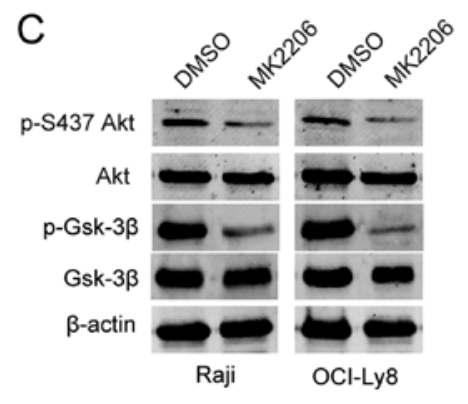

B
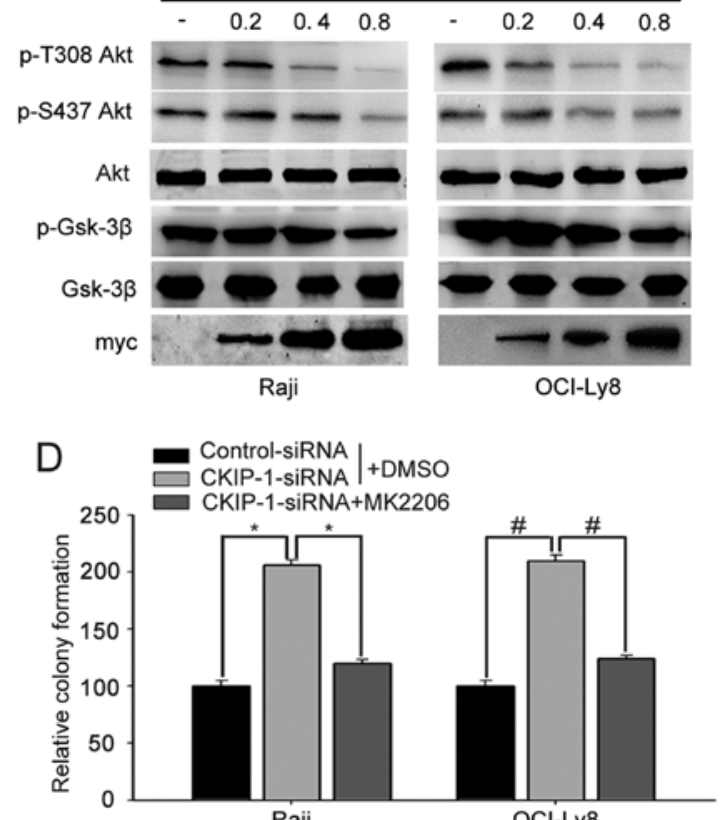



Figure 5. CKIP-1 promoted cell proliferation and inhibited apoptosis by enhancing phospho-AKT expression. (A) Endogenous protein-protein interaction between CKIP-1 and AKT was evaluated by immunoprecipitation (IP) with anti-CKIP-1 antibody followed by western blot analysis in OCI-Ly8 cells Control-IgG were used as a negative control for immunoprecipitation. (B) Raji and OCI-Ly8 cells were transfected with myc-CKIP-1 (0, 0.2, 0.4 and $0.8 \mathrm{mg}$ ). After transfection for $24 \mathrm{~h}$, cells were lysed and analysed for AKT expression by western blotting. (C) OCI-Ly8 and Raji cells were treated with AKT inhibitor MK2206 or DMSO (control), then analysed for indicated antibodies expression by western blotting. (D) Raji and OCI-ly8 cells were transfected with either myc-control or myc-CKIP-1, and treated with AKT inhibitor MK2206 or DMSO (control); then soft agar colony assays were used to evaluate cell proliferation. (E) Raji and OCI-ly8 cells were transfected with either CKIP-1-siRNA or myc-CKIP-1, or their respective control, then treated with MK2206 or DMSO, and flow cytometric analysis was used to analyse cell apoptosis. ${ }^{*}, \mathrm{P}<0.05$.

\section{Discussion}

Non-Hodgkin's lymphoma (NHL) comprises a heterogeneous group of lymphoproliferative disorders containing indolent as well as aggressive subtypes (1-3). The incidence of NHL was increased in recent years and is still associated with significant mortality. Despite intensive efforts in developing new therapies, the emergence of clinical drug resistance remains a barrier to successful treatment of lymphoma (6). Previous studies have shown that NHL cells adhered to FN or stromal cells (HS-5 cells) confer a multidrug-resistant phenotype and that disruption of cell adhesion-mediated signaling may increase the efficacy of chemotherapy drugs $(20,21)$.

CKIP-1, also known as PLEKHO1, the PH domaincontaining casein kinase 2 interacting protein-1, has been identified to play an important role in regulating many processes such as involved in tumor cell proliferation, muscle cell differentiation and cell apoptosis (8). In many cancers, the expression of CKIP-1 is markedly declining, including human osteosarcoma, colon (11) and breast cancer (15). Knockdown of CKIP-1 in macrophages can shorten the G1 phase and accelerate cell proliferation (13). In human colon cancer, CKIP-1 functions as a potential tumor suppressor and scaffold molecule that may modify and regulate the activities of several signaling pathways, including PI3K/mTOR and TGF- $\beta / \mathrm{BMP}$ (11). Furthermore, CKIP-1 enhances the sensitivity to chemotherapy drugs by targeting the Akt $\mathrm{PH}$ domain and suppressing Akt kinase activity in human cancers (8). Although CKIP-1 has been reported to reduce cell proliferation as a tumor suppressive gene in various cancers $(8,10,15,22)$, the biological role of CKIP-1 in the formation and progression of NHL remains unknown. 

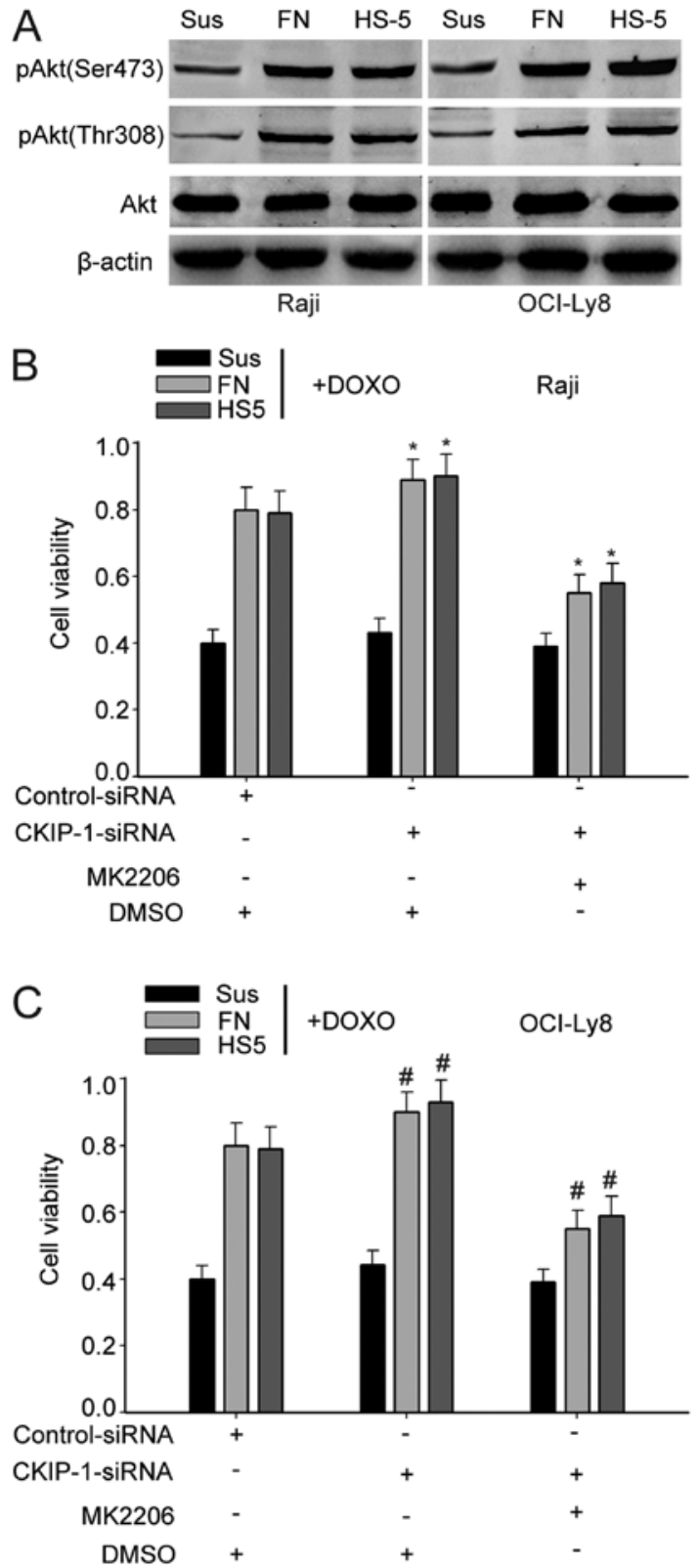

Figure 6. CKIP-1 reverses CAM-DR by regulating phosphorylation of Akt at Ser473 and Thr308. (A) Western blot analysis was performed to analyze the phosphorylation levels of Akt (Ser473 and Thr308). (B and C) Raji and OCILy8 cells were transfected with either siRNA-control or CKIP-1-siRNA, then treated with MK2206 or DMSO, then cells were allowed to adhere to FN or HS-5 cells or cultured in suspension along with addition of $1 \mu \mathrm{M}$ Doxo. Then, CCK-8 assays were performed to verify effects of CKIP-1-siRNA and MK2206 on CAM-DR in Raji and OCI-Ly8 cells. ${ }^{*}, \mathrm{P}<0.05$.

In the present study, we investigated the function of CKIP-1 in NHL for the first time. Firstly, we demonstrated that CKIP-1 was highly associated with NHL cell proliferation, and knockdown of CKIP-1 could promote the proliferation of NHL cells (Fig. 2C-F). Then, we determined the mechanism underlying the effect of CKIP-1 on regulating cell proliferation, and flow cytometric analysis revealed that knockdown of CKIP-1 generate a decrease in the percentage of cells in the G1 phase, but more cells were found in the G1 phase in the presence of myc-CKIP-1 (Fig. 3A and B). These findings revealed that the arrest in G1 phase might be the major reason for its inhibition of proliferation after
CKIP-1 was overexpressed in NHL cells. Moreover, we detected the expression levels of mRNA transcripts encoding cell cycle-regulatory proteins in NHL cell. OCI-Ly8 and Raji cells transfected with CKIP-1-siRNA expressed more c-Myc, cyclin D1 mRNA and cyclin E mRNA compared with control-siRNA transfected ones (Fig. 3C).

It has been widely demonstrated that CAM-DR through direct cell contact and adhesion may be a major cause of tumor cell drug resistance in hematologic malignancies (23-25). The present study showed that adhesion to HS-5 cells or FN decreased CKIP-1 expression, and the depressed levels of CKIP-1 significantly promote the proliferation of NHL cells (Fig. 2C-F). We found that knockdown of CKIP-1 could promote CAM-DR phenotype in NHL (Fig. 4D and E). Therefore, the apparent opposing roles of CKIP-1 suggest that, transient upregulation of CKIP-1 prior to treatment with chemotherapy may have a therapeutic benefit. However, how adhesion to FN or HS-5 cells influences CKIP-1 expression, as well as the molecular mechanisms involved, is still unclear and needs to be further elucidated.

For this reason, we elucidated the downstream target of CKIP-1 in regulating NHL cell tumorigenesis. In this regard, a previous study has identified that CKIP-1 interacts with Akt, and suppresses Akt phosphorylation and decreases Akt kinase activity in many human cancers (10). It has been reported that the PI3K/Akt signaling is the major pathway controlling cell growth and survival, and the elevated phosphorylated Akt levels could potentially be causative for resistance to chemotherapy $(26,27)$. Thus, we verified the role of PI3K/Akt in cell adhesion model. Western blot analysis revealed that the phosphorylation levels of Akt (Ser473 and Thr308) was more obvious in cells adhered to FN or HS-5 cells than cells cultured in suspension, suggesting that PI3K/Akt signaling pathways are involved in drug resistance (Fig. 6A). Moreover, Raji and OCI-Ly8 cells were transfected with myc-CKIP-1 in different doses. We found that knockdown of CKIP-1 markedly upregulated the phosphorylated Akt levels at Thr308 and Ser473 and the phosphorylation of GSK-3 $\beta$ in a dose-dependent fashion (Fig. 5B). Consequently, we detected the levels of apoptosis in CKIP-1 silencing cells and found that knockdown of CKIP-1 decreased cell apoptosis in the cell adhesion model, which can be reversed by Akt inhibitors (Fig. 6C and D). In summary, all data suggested that knockdown of CKIP-1 could accelerate proliferation and CAM-DR primarily through regulating the phosphorylation levels of Akt in NHL.

In conclusion, the present study suggests that CKIP-1 functions as a potential tumor suppressor and was highly associated with cell proliferation and played an important role in CAM-DR in NHL. Knockdown of CKIP-1 could accelerate the proliferation of NHL cells and promote CAM-DR phenotype in NHL. In the future, this may be an effective strategy for treatment of NHL.

\section{Acknowledgements}

The present study was supported by grants from the National Natural Science Foundation of China (nos. 81372537, 81570187 and 81070400), the Nantong University Foundation of Graduate Students of Science and Technology Innovation Project (no. YKC15053), the Medical Innovation Team and the 
Leading Talent Project of Jiangsu Province (LJ201136) and the Social Science and Technology Innovation and Demonstration in Nantong-Clinical Medical Science and Technology Project (HS2013067).

\section{References}

1. Harris NL, Jaffe ES, Stein H, Banks PM, Chan JK, Cleary ML, Delsol G, De Wolf-Peeters C, Falini B, Gatter KC, et al: A revised European-American classification of lymphoid neoplasms: A proposal from the International Lymphoma Study Group. Blood 84: 1361-1392, 1994.

2. Maxwell SA and Mousavi-Fard S: Non-Hodgkin's B-cell lymphoma: Advances in molecular strategies targeting drug resistance. Exp Biol Med (Maywood) 238: 971-990, 2013.

3. Shankland KR, Armitage JO and Hancock BW: Non-Hodgkin lymphoma. Lancet 380: 848-857, 2012.

4. Yagi K, Yamamoto K, Umeda S, Abe S, Suzuki S, Onishi I, Kirimura S, Fukayama M, Arai A, Kitagawa M, et al: Expression of multidrug resistance 1 gene in B-cell lymphomas: Association with follicular dendritic cells. Histopathology 62: 414-420, 2013.

5. Lwin T,Hazlehurst LA, Dessureault S, Lai R, Bai W, Sotomayor E, Moscinski LC, Dalton WS and Tao J: Cell adhesion induces p27 ${ }^{\mathrm{Kipl}}$-associated cell-cycle arrest through down-regulation of the $\mathrm{SCF}^{\mathrm{Skp} 2}$ ubiquitin ligase pathway in mantle-cell and other non-Hodgkin B-cell lymphomas. Blood 110: 1631-1638, 2007.

6. Lwin T, Zhao X, Cheng F, Zhang X, Huang A, Shah B, Zhang Y, Moscinski LC, Choi YS, Kozikowski AP, et al: A microenvironment-mediated c-Myc/miR-548m/HDAC6 amplification loop in non-Hodgkin B cell lymphomas. J Clin Invest 123: 4612-4626, 2013.

7. Mraz M, Zent CS, Church AK, Jelinek DF, Wu X, Pospisilova S, Ansell SM, Novak AJ, Kay NE, Witzig TE, et al: Bone marrow stromal cells protect lymphoma B-cells from rituximab-induced apoptosis and targeting integrin $\alpha-4-\beta-1$ (VLA-4) with natalizumab can overcome this resistance. Br J Haematol 155: 53-64, 2011.

8. Nie J, Liu L, He F, Fu X, Han W and Zhang L: CKIP-1: A scaffold protein and potential therapeutic target integrating multiple signaling pathways and physiological functions. Ageing Res Rev 12: 276-281, 2013.

9. Zhang L, Xing G, Tie Y, Tang Y, Tian C, Li L, Sun L, Wei H, $\mathrm{Zhu} \mathrm{Y}$ and He F: Role for the pleckstrin homology domaincontaining protein CKIP-1 in AP-1 regulation and apoptosis. EMBO J 24: 766-778, 2005.

10. Tokuda E, Fujita N, Oh-hara T, Sato S, Kurata A, Katayama R, Itoh T, Takenawa T, Miyazono $\mathrm{K}$ and Tsuruo $\mathrm{T}$ : Casein kinase 2-interacting protein-1, a novel Akt pleckstrin homology domain-interacting protein, down-regulates PI3K/Akt signaling and suppresses tumor growth in vivo. Cancer Res 67: 9666-9676, 2007.

11. Nie J, Liu L, Xing G, Zhang M, Wei R, Guo M, Li X, Xie P, Li L, He F, et al: CKIP-1 acts as a colonic tumor suppressor by repressing oncogenic Smurf1 synthesis and promoting Smurf1 autodegradation. Oncogene 33: 3677-3687, 2014.

12. Vilk G, Saulnier RB, St Pierre R and Litchfield DW: Inducible expression of protein kinase CK2 in mammalian cells. Evidence for functional specialization of CK2 isoforms. J Biol Chem 274 14406-14414, 1999.
13. Zhang L, Wang Y, Xiao F, Wang S, Xing G, Li Y, Yin X, Lu K, Wei R, Fan J, et al: CKIP-1 regulates macrophage proliferation by inhibiting TRAF6-mediated Akt activation. Cell Res 24: 742-761, 2014.

14. Wang Y,Liu F, Mao F, Hang Q, Huang X, He S, Wang Y, Cheng C, Wang $\mathrm{H}, \mathrm{Xu} \mathrm{G}$, et al: Interaction with cyclin $\mathrm{H} /$ cyclin-dependent kinase $7(\mathrm{CCNH} / \mathrm{CDK} 7)$ stabilizes $\mathrm{C}$-terminal binding protein 2 (CtBP2) and promotes cancer cell migration. J Biol Chem 288: 9028-9034, 2013

15. Zhang L, Tie Y, Tian C, Xing G, Song Y, Zhu Y, Sun Z and He F: CKIP-1 recruits nuclear ATM partially to the plasma membrane through interaction with ATM. Cell Signal 18: 1386-1395, 2006.

16. Canton DA, Olsten ME, Niederstrasser H, Cooper JA and Litchfield DW: The role of CKIP-1 in cell morphology depends on its interaction with actin-capping protein. J Biol Chem 281: 36347-36359, 2006.

17. Hennessy BT, Smith DL, Ram PT, Lu Y and Mills GB: Exploiting the PI3K/AKT pathway for cancer drug discovery. Nat Rev Drug Discov 4: 988-1004, 2005.

18. Liu T, Fang Y, Zhang H, Deng M, Gao B, Niu N, Yu J, Lee S, Kim J, Qin B, et al: HEATR1 negatively regulates Akt to help sensitize pancreatic cancer cells to chemotherapy. Cancer Res 76: 572-581, 2016.

19. Perna D, Karreth FA, Rust AG, Perez-Mancera PA, Rashid M, Iorio F, Alifrangis C, Arends MJ, Bosenberg MW, Bollag G, et al: BRAF inhibitor resistance mediated by the AKT pathway in an oncogenic BRAF mouse melanoma model. Proc Natl Acad Sci USA 112: E536-E545, 2015.

20. Hazlehurst LA, Damiano JS, Buyuksal I, Pledger WJ and Dalton WS: Adhesion to fibronectin via $\beta 1$ integrins regulates p2 $7^{\text {kipl }}$ levels and contributes to cell adhesion mediated drug resistance (CAM-DR). Oncogene 19: 4319-4327, 2000.

21. Lwin T, Hazlehurst LA, Li Z, Dessureault S, Sotomayor E, Moscinski LC, Dalton WS and Tao J: Bone marrow stromal cells prevent apoptosis of lymphoma cells by upregulation of anti-apoptotic proteins associated with activation of NF-kappaB (RelB/p52) in non-Hodgkin's lymphoma cells. Leukemia 21: 1521-1531, 2007.

22. Zhang L, Tang Y, Tie Y, Tian C, Wang J, Dong Y, Sun Z and He F: The PH domain containing protein CKIP-1 binds to IFP35 and Nmi and is involved in cytokine signaling. Cell Signal 19: 932-944, 2007.

23. Li ZW and Dalton WS: Tumor microenvironment and drug resistance in hematologic malignancies. Blood Rev 20: 333-342, 2006.

24. Wang K, Jiang Y, Zheng W, Liu Z, Li H, Lou J, Gu M and Wang X: Silencing of human phosphatidylethanolamine-binding protein 4 enhances rituximab-induced death and chemosensitization in B-cell lymphoma. PLoS One 8: e56829, 2013.

25. Nakagawa $Y$, Nakayama $H$, Nagata $M$, Yoshida $R$, Kawahara $K$, Hirosue A, Tanaka T, Yuno A, Matsuoka Y, Kojima T, et al: Overexpression of fibronectin confers cell adhesion-mediated drug resistance (CAM-DR) against 5-FU in oral squamous cell carcinoma cells. Int J Oncol 44: 1376-1384, 2014.

26. Bai H, Li H, Li W, Gui T, Yang J, Cao D and Shen K: The PI3K/AKT/mTOR pathway is a potential predictor of distinct invasive and migratory capacities in human ovarian cancer cell lines. Oncotarget 6: 25520-25532, 2015.

27. Liu M, Qi Z, Liu B, Ren Y, Li H, Yang G and Zhang Q: RY-2f, an isoflavone analog, overcomes cisplatin resistance to inhibit ovarian tumorigenesis via targeting the PI3K/AKT/mTOR signaling pathway. Oncotarget 6: 25281-25294, 2015. 